\title{
1918 : UNE VICTOIRE NAVALE EN CLAIR-OBSCUR
}

\author{
Jean de Préneuf, Thomas Vaisset
}

Comité d'études de Défense Nationale | «Revue Défense Nationale »

$2019 / 1 \mathrm{~N}^{\circ} 816$ | pages 42 à 47

ISSN 2105-7508

ISBN 9782919639823

DOI 10.3917/rdna.816.0042

Article disponible en ligne à l'adresse :

https://www.cairn.info/revue-defense-nationale-2019-1-page-42.htm

Distribution électronique Cairn.info pour Comité d'études de Défense Nationale.

(c) Comité d'études de Défense Nationale. Tous droits réservés pour tous pays.

La reproduction ou représentation de cet article, notamment par photocopie, n'est autorisée que dans les limites des conditions générales d'utilisation du site ou, le cas échéant, des conditions générales de la licence souscrite par votre établissement. Toute autre reproduction ou représentation, en tout ou partie, sous quelque forme et de quelque manière que ce soit, est interdite sauf accord préalable et écrit de l'éditeur, en dehors des cas prévus par la législation en vigueur en France. Il est précisé que son stockage dans une base de données est également interdit. 


\title{
1918 : une victoire navale en clair-obscur
}

\author{
Jean de Préneuf - Thomas Vaisset \\ Maître de conférences en histoire contemporaine \\ (Service historique de la Défense - Université de Lille). \\ Agrégé et docteur en histoire (Service historique de la \\ Défense).
}

$\mathrm{L}$ a Marine nationale a apporté une contribution significative à la signature de l'armistice du 11 novembre 1918. Avant tout parce qu'elle a participé à l'acquisition de la maîtrise de la mer, préalable indispensable à la victoire dans le cadre d'un conflit global et prolongé. Au plus fort des grandes batailles sur le continent (Verdun, la Somme...), le contrôle des routes maritimes a permis d'éviter l'effondrement du front occidental. Il a ensuite permis l'acheminement du corps expéditionnaire américain en avril 1917, prélude indispensable à la victoire finale. L'arrivée, saines et sauves, des armées de "l'Oncle Sam " a en effet provoqué le basculement définitif du rapport de force à l'Ouest. Mais la renonciation de Berlin a aussi été précipitée par la défection de ses alliés austro-hongrois et ottoman qui ont été vaincus grâce à une stratégie périphérique dont l'importance est trop souvent minorée. Or, les coups de boutoir des armées de l'Entente en Italie et sur les fronts oriental et moyen-oriental n'ont été rendus possibles, ici aussi, que par la victoire dans la lutte anti-sous-marine (ASM) en Méditerranée dont $50 \%$ des moyens sont alors mis en œuvre par la Marine nationale. Ce succès est en effet la condition sine qua non de la projection et du soutien des corps expéditionnaires. Enfin, les arsenaux de la Marine ont joué un rôle décisif lors de la première moitié du conflit jusqu'à ce que la mise en place d'une économie de guerre, dès 1916-1917, ne vienne relativiser leur part - qui reste néanmoins cruciale - dans la mobilisation industrielle.

Victorieuse, la Marine de 1918 l'est donc assurément. Mais il s'agit d'une victoire à la Pyrrhus aussi paradoxale et coûteuse qu'elle est incertaine et amère. La contribution majeure au succès final a été réalisée au prix d'une utilisation intensive de l'outil naval de 1914. Elle a été aussi permise par un profond renouvellement, que ce soit en surface, sous l'eau, dans les airs ou sur terre. Or, cette refondation reste partielle car il a fallu donner la priorité aux opérations terrestres. Achever la modernisation en cours de la flotte et des arsenaux représente aussi une gageure, car le pays est exsangue financièrement. Traumatisée par le sacrifice des Poilus dans les tranchées, la majorité de la population

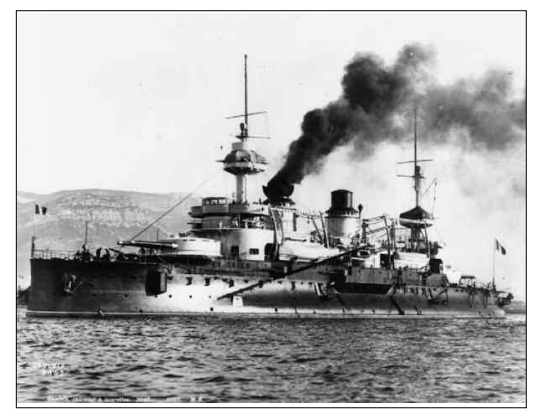

Le Gaulois, torpillé en 1916 par un U-Boote. 
et des élites aspire à toucher les dividendes de la paix qui se profile et comprend mal le rôle joué par la marine comme la nécessité de poursuivre son adaptation aux mutations de la guerre sur mer. Cette incompréhension est d'autant plus largement partagée que la situation stratégique apparaît favorable avec la disparition des menaces navales italienne, austro-hongroise et surtout allemande. Dresser le portrait de la marine à la fin 1918, c'est donc présenter le visage d'une flotte entre deux rives, entre paix et guerre, entre obsolescence et modernisation, entre victoire à portée de main et doutes face à un avenir incertain.

\section{L'armistice n'est pas la fin de la guerre}

Sur mer comme à terre, la Grande Guerre ne s'achève pas le 11 novembre 1918. Les unités de la Marine continuent d'assurer le blocus afin de faire pression sur l'Allemagne jusqu'à la signature de la paix, le 28 juin 1919, à Versailles. Des forces importantes sont aussi positionnées devant les côtes de Méditerranée orientale, de Constantinople au Levant. Mais c'est en mer Noire que le dispositif est le plus important. Engagée pour surveiller l'évacuation des troupes allemandes après l'armistice de Brest-Litvosk, en mars 1918, puis pour soutenir les troupes contrerévolutionnaires et alliées qui luttent contre les Bolchéviques, l'Escadre de la mer Noire, commandée par le vice-amiral Amet, rencontre de nombreuses difficultés. À la complexité de la situation politique s'ajoute la défaillance du ravitaillement et des communications. La tension monte au sein des équipages qui veulent regagner au plus vite leurs foyers et des mutineries éclatent en avril 1919. Le climat est lourd également dans les arsenaux métropolitains dont les 64000 ouvriers aspirent au retour à la normale après quatre années de travail dans des conditions très rudes. Une partie des ouvriers et des équipages se montre aussi sensible à la propagande révolutionnaire. Des mutineries, à caractère d'abord politique, finissent par éclater en juillet 1919, à l'instar de ce qui se produit dans la plupart des grandes flottes.

\section{Entre obsolescence et adaptation inachevée au nouveau visage de la guerre sur mer}

Au-delà de leur dimension sociale et politique plus ou moins marquée, ces troubles trahissent également l'ampleur du déclassement et du délabrement de la marine à l'issue de plus de quatre années de conflit. Au jour de l'armistice, la flotte française comprend environ 650000 tonnes de bâtiments en service. Mais les hommes sont épuisés, les matériels usés et souvent obsolètes.

L'absolue priorité du soutien des opérations terrestres a conduit à interrompre le renouvellement de la flotte de combat. La Marine nationale n'a lancé aucun cuirassé ou croiseur moderne depuis 1914. Les seules unités construites dans les arsenaux ont été destinées à la lutte ASM, construction complétée par l'achat de plusieurs centaines de bâtiments légers à l'étranger. Dans ces conditions, comme 
en 1914, la Marine française reste une flotte d'échantillons, l'ensemble est hétérogène et sa valeur militaire très inégale.

Les hostilités ont en effet révélé l'inadaptation de la flotte de 1914 aux nouvelles conditions de la guerre navale. Un effort important de modernisation a été entrepris, mais il reste inachevé sur de nombreux points. Si, en novembre 1918, la flotte aligne 1131 unités de tout tonnage affectées à la lutte ASM, seule une minorité de celles-ci est dotée de l'hydrophone inventé par Paul Langevin, un appareil de détection à la pointe de la modernité. Encore embryonnaire en 1914, l'aéronautique maritime représente désormais près du dixième des effectifs de la Marine nationale et met en ouvre 1264 hydravions et avions, ainsi que 37 dirigeables. Quelques mois à peine après la Royal Navy, la Marine nationale vient de procéder au premier lancement d'avion depuis le pont d'un cuirassé. Après les premières tentatives d'essais réalisés sur la Foudre à la veille de la guerre, il s'agit là des premiers balbutiements de l'aéronavale embarquée. Il faudra néanmoins attendre le début des années 1920 pour que soit mis sur cale le premier porte-avions français, le Béarn, dont les qualités militaires sont relatives. Cet exemple montre que l'effort d'innovation est autant technique que doctrinal. Alors qu'en 1914, les aéronefs étaient d'abord conçus comme des systèmes permettant le réglage d'artillerie et l'éclairage, ils servent désormais aussi au bombardement de navires et de sousmarins, même si l'efficacité de cette dernière mission reste très limitée. Autre " innovation " tactique apparue au cours du conflit, la redécouverte et le perfectionnement du système des convois dans lesquels la Marine nationale a joué un rôle moteur. De façon plus générale, c'est toute l'intégration des différentes composantes de la Marine dans la conduite des opérations qui paraît beaucoup plus poussée en 1918 qu'en 1914.

\section{Recul relatif de l'outil naval}

Mais, si l'effort de modernisation est bien réel, il n'en reste pas moins que l'outil naval français a connu un déclin relatif entre 1914 et 1918. En quatre ans, la marine italienne a achevé, ou mis sur cale, six cuirassés et deux croiseurs (ainsi que trente-deux contre-torpilleurs, trente-sept torpilleurs et soixante-dix-sept sousmarins). Le rapport de force en Méditerranée semble s'inverser et compromettre la sécurité des communications maritimes de la France avec l'Afrique du Nord et le Levant. De leur côté, Britanniques et Américains ont lancé de gigantesques programmes de construction navale. Paris se trouve donc en situation délicate lors des négociations des aspects navals de l'armistice et à l'heure de discuter des modalités de la paix sur mer. Poursuivre la modernisation à peine esquissée pendant le conflit s'impose si la Marine nationale entend préserver son rang et la France peser à Versailles. Or, la remise à niveau de la flotte s'annonce problématique. Le premier obstacle réside dans les limites de l'infrastructure industrielle dues au sousinvestissement chronique pendant le conflit. Il faut aussi gérer le difficile processus de "renavalisation " d'arsenaux qui ont privilégié la satisfaction des besoins de 
l'Armée. En novembre 1918, à peine le tiers du personnel des arsenaux travaille à des tâches relevant de la construction ou de la réparation des navires de guerre. Les ports militaires français peinent à assurer l'entretien des bâtiments, et il faut faire appel aux chantiers privés et aux arsenaux alliés. Le second obstacle à la poursuite de la modernisation de la Marine est budgétaire et politique, car la priorité va à la reconstruction du pays. La pénurie de crédits conduit une partie des établissements industriels de la Marine à travailler temporairement au profit du secteur civil pour alimenter leur plan de charge. Et, dès 1919, le tonnage de la flotte retombe à 485000 tonnes.

\section{Un avenir incertain et une pointe d'amertume}

Débats doctrinaux et stratégiques viennent s'ajouter à l'incertitude politique qui règne quant à l'avenir de la Marine au moment de la signature de l'armistice. Il faut repenser l'utilisation de l'arme sous-marine en même temps que l'intégration de l'aéronavale dans les flottes. Se pose aussi la question du très coûteux renouvellement des corps de bataille, alors même que les peuples aspirent au développement de l'État social, à la garantie de la paix par des moyens non militaires ou l'institutionnalisation de la sécurité collective, que ce soit l'utilisation de l'arme monétaire ou la création de la Société des nations. L'incertitude est donc grande et les débats font rage, arbitrés par les opinions publiques et les puissances alliées dont Paris, affaibli financièrement et à la recherche de garanties de sécurité, dépend en partie.

L'apparition des $U$-Boote a ébranlé les fondements de la stratégie navale tels que l'amiral américain Alfred Mahan les avait posés, en contestant la primauté du cuirassé en tant que capital ship au sein de la composition des flottes. La véritable surprise tactique consiste bien plus dans les succès remportés par les sous-marins contre les bâtiments militaires de surface que dans une guerre de course que les $U$-Boote n'ont fait que remettre au goût du jour. Ce constat inspire une partie des officiers qui songent à refonder la Marine autour d'une force sous-marine océanique. Elle constituerait un outil dissuasif à l'égard du commerce britannique et ferait peser une menace certaine à l'égard des escadres de la Royal Navy et de l'US Navy

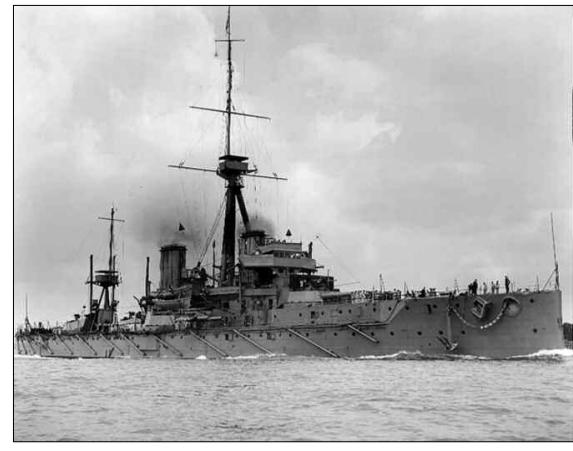

HMS Dreadnought, 1906. qui dominent les océans à la fin de la guerre. Pourtant, au lendemain du conflit, les adversaires de l'arme sous-marine sont nombreux et pointent la « faillite " d'un système d'arme jugé encore peu performant, surtout face au développement des moyens de lutte ASM au cours du conflit. Comme leurs pairs américains et britanniques, la majorité des amiraux français entend fin 1918 donner la priorité au renouvellement du corps de bataille en relançant un ambitieux programme de cuirassés de la famille des super-dreadnoughts, 
complété par la construction de croiseurs et de flottilles aériennes et de surface pour les escorter et les éclairer.

Objet de débat au sein de la Marine, le sous-marin est aussi et surtout au cœur d'intenses tractations diplomatiques. Elles opposent les puissances favorables à son interdiction et celles qui entendent libéraliser son emploi. Les marines militaires et marchandes britanniques ont été durement éprouvées par les U-Boote. Londres plaide donc en faveur d'une mise hors-la-loi de cette arme nouvelle, d'autant plus que le gouvernement de Sa Majesté se trouve sous la pression d'une opinion publique profondément heurtée par les pertes civiles provoquées par la guerre sousmarine à outrance et le blocus. En revanche, Paris se place, comme Rome, dans la logique du faible au fort et voit dans le sous-marin une arme de dissuasion qui doit permettre d'écarter le risque de guerre. Cantonnée en France au cercle des experts et des états-majors, cette divergence d'appréciation entre alliés apparaît dès 1918.

De même, à l'heure de l'armistice, le débat sur l'opportunité de la relance de l'effort budgétaire à consentir pour moderniser la flotte reste pour le moment feutré et embryonnaire. La France possède-t-elle encore les moyens nécessaires pour nourrir une véritable ambition navale alors qu'elle n'a plus d'ennemi ? La marine italienne est désormais une alliée, l'essentiel de la flotte allemande se saborde à Scapa Flow, en juin 1919, et ce qu'il en reste est cantonné au rôle de garde-côte en Baltique. Il n'y a là, rien qui puisse inciter les dirigeants français à mettre l'outil naval au premier rang de leurs préoccupations, surtout quand la reconstruction des régions dévastées du Nord-Est prime. La méconnaissance de l'ampleur de la contribution de la Marine à la victoire ne fait que renforcer ce relatif désintérêt des élus. Significativement, "l'Armée de mer " n'est pas mentionnée dans la loi du 17 novembre 1918 qui proclame que, "les Armées et leurs chefs [...] ont bien mérité de la Patrie ». L'oubli n'est réparé que le 4 décembre, mais, seulement par le vote d'une simple motion de félicitations de la part de la Commission parlementaire de la Marine. Au-delà des élus, l'absence de victoire décisive dans un combat d'escadre identifié tranche aux yeux de l'opinion publique avec les succès de la Marne et de Verdun. Elle peine à prendre la mesure de l'ampleur tant des sacrifices que de l'effort d'adaptation auquel la Marine nationale a dû consentir. Dans un pays traumatisé par l'hécatombe des Poilus, les 11500 marins disparus au combat représentent moins de $1 \%$ des pertes totales françaises. Dans les mémoires domine le souvenir des fusiliers marins de l'amiral Ronarc'h, qui se sont sacrifiés pour enrayer l'offensive allemande à Dixmude en novembre 1914 et qui sont les seuls marins invités à participer au défilé de la Victoire le 14 juillet 1919.

Dans un conflit prolongé aux dimensions planétaires, la puissance maritime a fait sentir tous ses effets. Si la guerre a démontré le caractère illusoire du navalisme mahanien sans nuance qui domine en 1914, elle a confirmé toute l'importance du contrôle de la mer, cher à Sir Julian Corbett. Comme le théorise en France l'amiral Raoul Castex à l'issue du conflit, ces quatre années ont aussi apporté la preuve qu'il n'est de stratégie que globale et s'appuyant sur la liaison interarmées. 
Pour autant, la contribution de la Marine nationale à la victoire reste en partie mal connue ou incomprise. Elle peine à cristalliser dans une opinion publique très marquée par les pertes connues par les Poilus dans les tranchées. Dans ces conditions, le défi est de convaincre politiques et société civile de la nécessité de poursuivre et accélérer l'effort de modernisation ébauché pendant le conflit, sous peine de ne pouvoir peser dans les arbitrages sur l'après-guerre. Déjà perceptibles lors de la négociation des clauses navales de l'armistice, tensions et divergences avec les autres vainqueurs de la guerre éclatent au grand jour lors de la conférence de Washington en 1922. Lapproche britannique quant à la guerre sousmarine s'y impose et une stricte limitation des constructions navales est instaurée selon un système de quotas particulièrement favorable aux États-Unis et à la Grande-Bretagne. Quant à la rue Royale, elle entend tirer les leçons de cette séquence délicate. Désormais, l'un des axes majeurs de sa politique est de convaincre les élus et la société civile de la nécessité, pour la France, d'avoir une véritable ambition navale. Ce n'est pas le moindre enseignement du conflit.

NDLR : article initialement paru dans le numéro hors-série «La Marine dans la Grande Guerre », Études Marines, sous le titre " Une marine victorieuse, mais une victoire... à la Pyrrhus ".

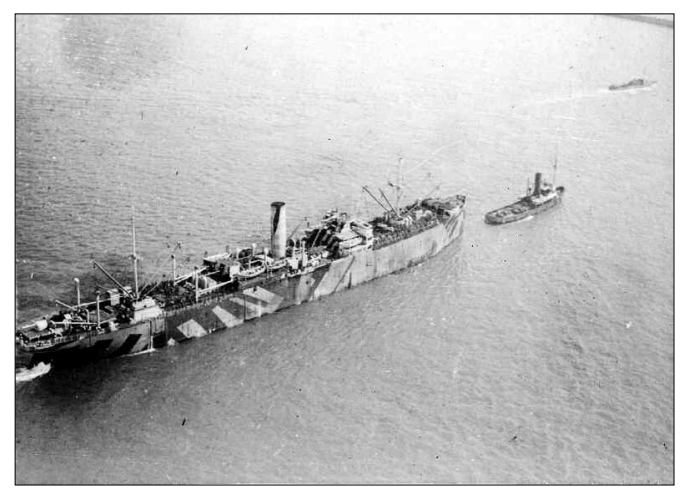

Source : SHD, iconothèque Air.

Un cargo à proximité du port du Havre en 1918. On remarque le camouflage de l'unité, un art qui connut de grands progrès au cours du conflit, destinée à rendre son repérage plus ardus pour les $U$-Boote. Le transport des troupes et le ravitaillement du pays sont des contributions essentielles, mais méconnues, de la Marine nationale à la victoire finale du pays au cours de la Grande Guerre.

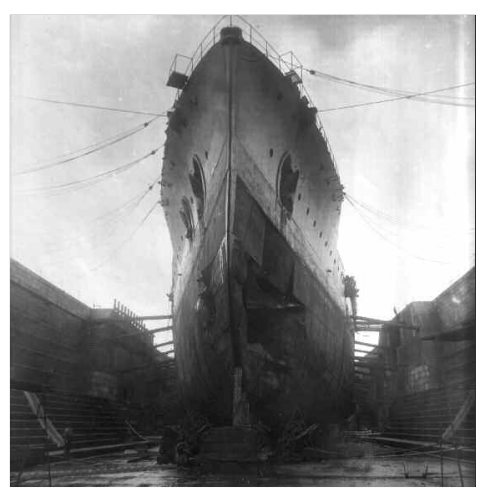

Source : SHD Marine, fonds privé Chopart.

Le cuirassé Jean Bart en cale sèche à Malte. Portant la marque du commandant en chef de l'Armée navale, le bâtiment a été torpillé dans le canal d'Otrante dans la nuit du 21 décembre 1914. Unité récente et bien compartimentée, le Jean Bart est parvenu, en dépit des dégâts, à gagner la base anglaise.

\section{ÉLÉMENTS DE BIBLIOGRAPHIE}

Jean de Préneuf, Thomas Vaisset et Philippe Vial (dir.) : « La Marine nationale et la Première Guerre mondiale : une histoire à redécouvrir ", Revue d'histoire maritime, n² 20, PU Paris-Sorbonne, mai 2015 ; "La Marine dans la Grande Guerre », Études marines, hors-série, CESM, novembre 2018.

Paul G. Halpern : A Naval History of World War I [1994] ; Londres-New York, Routledge, 1995.

Lawrence Sondhaus : The Great War at Sea. A Naval History of the First World War ; Cambridge, Cambridge University Press, 2014. 